\title{
NON SPECIFIC CAECAL ULCER (NSCU) - AN OPPORTUNITY TO EXPLORE A RARE CASE CLINICALLY PRESENTED WITH ONE OF THE COMMON ABDOMINAL PRESENTATION
}

K. L. Narasimha Rao, S. S. R. Nagendra Babu, G. Kiran, N. Mohan Rao, V. V. Durga Prasad
1. Associate Professor. Department of General Surgery, GSL Medical College.
2. Post Graduate. Department of General Surgery, GSL Medical College.
3. Professor \& HOD. Department of General Surgery, GSL Medical College.
4. Post Graduate. Department of General Surgery, GSL Medical College.

\section{CORRESPONDING AUTHOR:}

Dr.K.L.Narasimha Rao,

Associate professor,

Department of General Surgery,

GSL Medical college.Laxmipuram,NH5,

Rajanagaram Mandal. Rajahmundry-533296.

E-mail: klnarasimharao@hotmail.com

\begin{abstract}
A case of 42 years old male patient clinically suggestive of peritonitis due to appendicular perforation underwent exploratory laparotomy revealed normal appendix along with a large caecal perforation without any underlying primary or secondary pathologies. Caecostomy with Malecot's catheter with purse string sutures around the catheter was put in place. The suspicion of inadequate differential diagnosis later on revealed it as non specific caecal ulcer (NSCU) in the histopathology sample of perforated ulcer.
\end{abstract}

KEY WORDS: Non specific caecal ulcer.

CASE PRESENTATION: This is a case of non specific caecal ulcer (NSCU) of the large intestine. NSCU has no specific presentation features and it can mimic host of conditions like appendicitis, diverticulosis and cancer colon. There are only few cases where a specific pre operative diagnosis was made. The number of NSCU being reported recently is less. This is due to advent of newer diagnostic techniques to identify the etiology of these ulcers. Hence the term NSCU is no longer employed in current literature because a specific diagnosis is attained in most of the cases $^{1}$.

CLINICAL PRESENTATION: The present case of 42 year old male patient admitted in our department presented with abdominal distension pain, hypotension and decreased urine output. On physical observation distension with diffused tenderness, board like rigidity all over the abdomen along with umbilicus middle line eversion along with shifting of dullness, sluggish bowel sounds was observed.

INVESTIGATIONS: X-ray erect abdomen with domes revealed gas under right side of diaphragm. Diagnostic peritoneal lavage reveals $10 \mathrm{ml}$ of purulent ascitic fluid with fecal odour. Ultrasound reveals distended small bowel loops and free fluid in the abdomen. Blood examination confirmed leucocytosis, elevated blood urea and serum creatinine levels. His HIV and HBsAg tests were negative. Per rectal examination revealed collapsed lumen. The patient with preoperative diagnosis of hallow viscus perforation underwent exploratory laparotomy. 


\section{CASE REPORT}

INTRAOPERATIVE FINDINGS: Laparotomy revealed $6 \times 4 \mathrm{~cm}$ cecal perforation, multiple interloop adhesions filled with pus, multiple flakes and 1 liter of peritoneal fluid. Appendix found normal. Small bowel distended, large bowel collapsed, without any evidence of visible malignancies or strictures.

MANAGEMENT: Caecostomy- Malecot's catheter with purse string sutures around the catheter was put in place. Adenolysis was performed. Extensive peritoneal normal saline lavage of 3 liters was done and abdominal drainage was provided. The edges of the ulcer were sent to histopathology.

HISTOPATHOLOGY: The histopathology reported non specific ulcer of the caecum, no evidence of any granulomatous inflammation/amoebiasis/malignancy.

FOLLOWUP: Post surgery patient status and general condition improved in terms of normalized renal parameters and vitals. After repeated follow ups of weekly for first two weeks, monthly for next 3 months, and the patient recovered completely.

DISCUSSION: Caecal perforations are commonly encountered as a part and parcel of various associated disease processes, in accordance with Laplace's law. Laplace's law dictates that the intraluminal pressure needed to stretch the wall of a hollow tube is inversely proportional to its radius. The caecum has the largest diameter of the colon, and as such, requires the least amount of pressure to distend ${ }^{2-4}$ The diameter of the caecum in which perforation is imminent has been estimated to be between $9 \mathrm{~cm}$ and $16 \mathrm{~cm}^{5}$.Caecal perforations are usually seen associated with entities such as diverticular disease, inflammatory bowel diseases, closed loop obstructions ${ }^{6}$, pancreatic carcinomas ${ }^{7}$, colorectal cancers ${ }^{8}$, hirschsprung's disease ${ }^{9}$, etc, in the presence of a patent ileo-caecal valve. In dealing with an emergency colonic obstruction leading to a caecal perforation, few authors favour some form of primary anastomosis as opposed to others favoring creating of an initial stoma after a standard right hemi-colectomy.

The main clinical manifestations include abdominal pain mimicking appendicitis (50\%), lower gastrointestinal hemorrhage (33\%), perforation (19\%), and abdominal mass (16\%). The usual location of the ulcers is the caecum and ascending colon (67\%), then transverse, hepatic, and splenic flexures (18\%), and descending and sigmoid colon (15\%). The diagnosis is best established by colonoscopy. The nonoperative conservative management is probably indicated in the uncomplicated cases with follow-up colonoscopic studies to ensure complete healing. The etiology of this condition is still unknown ${ }^{10}$. Idiopathic ulcer of the caecum has been considered a rare condition requiring surgical treatment because of a high incidence of complications, including bowel perforation. Caecal ulcerations are usually diagnosed at the time of surgery for presumed appendicitis or peritonitis of unknown origin. Preoperative diagnosis of caecal ulcer by barium enema has been unreliable and previous literature cites only one case diagnosed by colonoscopy. If the diagnosis of caecal ulcer is made by colonoscopy in a patient without evidence of an acute abdomen, conservative management may be followed by complete healing, avoiding unnecessary surgery ${ }^{11}$.In the present case with acute presentation, surgery was inevitable which was performed and the histopathology confirmed the case to be non specific caecal ulcer. 
CONCLUSION: Usual occurrence of NSCU has reduced due to advent of sensitive diagnostic procedures in the recent times. It is usually presentation mimics many other large intestinal conditions. The present case as well with preliminary diagnosis of peritonitis due to appendicular perforation revealed large caecal perforation, the histopathology of the perforated edge conformed it as non specific caecal ulcer (NSCU).Usual common case presentations sometimes explored with suspicion can reveal rare cases.

\section{REFERENCES:}

1. Ram D, Karthikeyan VS, Sistla SC. Nonspecific Cecal Ulcer: An Obsolete Jargon. J Interdiscipl Histopathol. 2013, 1: 85-88.

2. Saegesser F, Chapuis G, Rausis C, Tabrizian M, Sandblom P: Intestinal distention and colonic ischemia: occlusive complications and perforations of colo-rectal cancers. A clinical application of Laplace's Law. Chirurgie 1974, 100:502-516.

3. Vanek VW, Al-Salti M: Acute pseudo-obstruction of the colon (Olgivie's syndrome): an analysis of 400 cases. Dis Colon Rectum 1986, 29:203-210.

4. Gierson ED, Storm FK, Shaw W, Coyne SK: Caecal rupture due to colonic ileus. Br J Surg 1975, 62:383-386.

5. Acea-Nebril B, Parajo-Calva A, Taboada-Filguira L, et al.: Surgical indications in acute pseudo-obstruction of colon (Ogilvie syndrome). Rev Esp Enferum Dig 1993, 84:1979.

6. Novy S, Rogers LF, Kirkpatrick W: Diastatic rupture of the cecum in obstructing carcinoma of the left colon. Radiographic diagnosis and surgical implications. Am J Roentgenol 1975, 123:281-286.

7. Tempia-Caliera AA, Horvath LZ, Zimmermann A, Tihanyi TT, Korc M, Friess H, Buchler MW: Adhesion molecules in human pancreatic cancer. J Sur Oncol 2002, 79:93-100.

8. Carraro PG, Segala M, Orlotti C, Tiberio G: Outcome of large-bowel perforation in patients with colorectal cancer. Dis Colon Rectum 1998, 41:1421-1426.

9. Yamamoto T, Hayashi Y, Suzuki H, et al.: Early onset of cecal perforation in neonatal, recto-sigmoid type Hirschsprung disease. Acta Pediatr Jpn 1994, 36:717-9.

10. Ona FV, Allende HD, Vivenzio R, Zaky DA, Nadaraja N. Diagnosis and management of nonspecific colon ulcer. Arch Surg. 1982 Jul;117(7):888-94.

11. Blundell CR, Earnest DL Idiopathic cecal ulcer. Diagnosis by colonoscopy followed by nonoperative management Dis Sci. 1980 Jul;25(7):494-503 